OPEN ACCESS

Edited by:

Christian Kirschneck, University Medical Center

Regensburg, Germany

Reviewed by:

Sabrina Kathrin Schulze,

University of Potsdam, Germany

Guido Artemio Marañón-Vásquez,

Federal University of Rio de Janeiro,

Brazil

*Correspondence:

Shao-yu Chen

shaoyu.chen@louisville.edu

Specialty section:

This article was submitted to

Cell Growth and Division,

a section of the journal

Frontiers in Cell and Developmental

Biology

Received: 16 July 2020

Accepted: 14 September 2020

Published: 02 October 2020

Citation:

Yuan F, Yun Y, Fan H, Li Y, Lu L,

Liu J, Feng $W$ and Chen S-y (2020) MicroRNA-135a Protects Against

Ethanol-Induced Apoptosis in Neural Crest Cells and Craniofacial Defects

in Zebrafish by Modulating

the Siah1/p38/p53 Pathway.

Front. Cell Dev. Biol. 8:583959.

doi: 10.3389/fcell.2020.583959

\section{MicroRNA-135a Protects Against Ethanol-Induced Apoptosis in Neural Crest Cells and Craniofacial Defects in Zebrafish by Modulating the Siah1/p38/p53 Pathway}


Wenke Feng ${ }^{1,2,4}$ and Shao-yu Chen ${ }^{1,2 *}$

'Department of Pharmacology and Toxicology, University of Louisville Health Sciences Center, Louisville, KY, United States, 2 University of Louisville Alcohol Research Center, Louisville, KY, United States, ${ }^{3}$ College of Environment and Resource, Research Center of Environment and Health, Shanxi University, Taiyuan, China, ${ }^{4}$ Department of Medicine, University of Louisville, Louisville, KY, United States

MicroRNAs (miRNAs) are small non-coding RNAs that are involved in various biological processes, including apoptosis, by regulating gene expression. This study was designed to test the hypothesis that ethanol-induced downregulation of miR-135a contributes to ethanol-induced apoptosis in neural crest cells (NCCs) by upregulating Siah1 and activating the p38 mitogen-activated protein kinase (MAPK)/p53 pathway. We found that treatment with ethanol resulted in a significant decrease in miR-135a expression in both NCCs and zebrafish embryos. Ethanol-induced downregulation of miR-135a resulted in the upregulation of Siah1 and the activation of the p38 MAPK/p53 pathway and increased apoptosis in NCCs and zebrafish embryos. Ethanol exposure also resulted in growth retardation and developmental defects that are characteristic of fetal alcohol spectrum disorders (FASD) in zebrafish. Overexpression of miRNA-135a significantly reduced ethanol-induced upregulation of Siah1 and the activation of the p38 MAPK/p53 pathway and decreased ethanol-induced apoptosis in NCCs and zebrafish embryos. In addition, ethanol-induced growth retardation and craniofacial defects in zebrafish larvae were dramatically diminished by the microinjection of miRNA-135a mimics. These results demonstrated that ethanol-induced downregulation of miR-135a contributes to ethanol-induced apoptosis in NCCs by upregulating Siah1 and activating the p38 MAPK/p53 pathway and that the overexpression of miRNA-135a can protect against ethanol-induced apoptosis in NCCs and craniofacial defects in a zebrafish model of FASD.

Keywords: miRNA-135a, ethanol, apoptosis, neural crest cells, Siah1, craniofacial defects, zebrafish

Abbreviations: miRNA, microRNA; FASD, fetal alcohol spectrum disorders; NCCs, neural crest cells; Siah1, seven in absentia homolog 1; Bcl-2, B-cell lymphoma 2; Nrf2, nuclear factor (erythroid-derived 2)-like 2; MAPK, mitogen-activated protein kinase; $3^{\prime}$-UTRs, $3^{\prime}$-untranslated region; hpf, hours post-fertilization; dpf, days post fertilization; TUNEL, terminal deoxynucleotidyl transferase dUTP nick end labeling; Bak, Bcl-2 homologous antagonist/killer; PUMA, p53 upregulated modulator of apoptosis; m, Meckel's cartilage; e, ethmoid plate; t, trabeculae cranii; pq, palatoquadrate; hs, hyosymplectic; ch, ceratohyal; cb, ceratobranchial; bh, basihyal. 


\section{INTRODUCTION}

Fetal alcohol spectrum disorder (FASD) is an umbrella term used to describe the range of disorders that occur in an individual whose mother drinks alcohol during pregnancy. Individuals with FASD may have abnormal facial features, growth retardation, central nervous system dysfunction, and learning disabilities (Mukherjee et al., 2006; Mantha et al., 2014). Studies have shown that the ethanol-induced apoptosis in neural crest cells (NCCs), a multipotent progenitor cell population that can give rise to a diversity of cell types, including mesenchymal cells that form craniofacial cartilages, bones, and dermis (Smith, 1997; Dash and Trainor, 2020), is one of the major components of the pathogenesis of FASD (Cartwright and Smith, 1995a; Muralidharan et al., 2013). Multiple signaling pathways have been reported to be involved in ethanol-induced apoptosis, including B-cell lymphoma 2 (Bcl-2) (Hong et al., 2002), p53 (Jana et al., 2010), nuclear factor (erythroid-derived 2)-like 2 (Nrf2) (Chen et al., 2013a), p38 mitogen-activated protein kinase (MAPK), and seven in absentia homolog 1 (Siah1) (Yuan et al., 2017).

Seven in absentia homolog 1 is a member of a highly conserved family of E3 ubiquitin ligases (Carthew and Rubin, 1990) that is widely expressed in mouse embryos and adult tissues (Della et al., 1993). Siah plays a critical role in a variety of biological processes, including apoptosis (Nemani et al., 1996). Our previous study has demonstrated that ethanol treatment can significantly increase the expression and nuclear translocation of Siah1 in NCCs and that Siah1 signaling plays a critical role in ethanol-induced apoptosis in NCCs (Sun et al., 2014). We have also shown that ethanol-induced upregulation of Siah1 can induce apoptosis in NCCs through p38 MAPK-mediated activation of the p53 signaling pathway (Yuan et al., 2017). However, the mechanisms by which ethanol upregulates Siah1 in NCCs are not clear.

MicroRNAs are small non-coding RNAs that are involved in various physiological and pathological processes, including apoptosis, by regulating gene expression. MiR-135a, a member of the miR-135 superfamily, has been reported to be involved in the tumorigenesis and act as a tumor suppressor (Wu et al., 2012; Kroiss et al., 2015; Xu et al., 2016). MiR-135a expression in astrocytes has also been linked to brain inflammation and angiogenesis in Alzheimer's disease (Ko et al., 2015). Studies have also shown that miR-135a can promote proliferation and inhibit apoptosis of astrocytes in the bacterial meningitis rat models (Dong et al., 2019). Overexpression of miR-135a can also protect human umbilical vein endothelial cells against mechanical stretch-induced increases in apoptosis and ventilatorinduced lung injury (Yan et al., 2018). In addition, Siah1 has been identified as a target of miR-135a in HeLa cells and mouse zygotes (Pang et al., 2011). It has been reported that miR-135a can upregulate $\beta$-catenin in cervical epithelial cells by targeting Siah 1 (Leung et al., 2014).

Zebrafish is a well-established animal model for biomedical research, including FASD research (Bilotta et al., 2004) and an excellent animal model to study gene-ethanol interactions (McCarthy et al., 2013). The rapid external development of zebrafish embryos coupled with their transparency allows rapid analysis of structure and function in the intact embryos (Beis and Stainier, 2006). Studies have demonstrated that embryonic exposure to ethanol resulted in dysmorphology and behavioral deficits that parallel those of FASD in zebrafish (Bilotta et al., 2004; Carvan et al., 2004; Fernandes and Gerlai, 2009; Wang et al., 2018) and that the developmental defects induced by embryonic ethanol exposure can be examined in zebrafish larvae by measuring the eye diameter (Bilotta et al., 2004), body length, and craniofacial cartilage (Marrs et al., 2010).

In the present study, an in vitro model of NCCs and a zebrafish model of FASD were used to elucidate the mechanisms by which miR-135a modulates ethanol-induced apoptosis in NCCs and craniofacial defects in zebrafish embryos. We found that ethanol treatment decreased the expression of miR-135a and thereby increased the expression of its direct target, Siah1, which, in turn, activated the p38 MAPK/p53 pathway, increased apoptosis in NCCs and zebrafish embryos and resulted in growth retardation and developmental defects. Overexpression of miR-135a significantly reduced ethanolinduced upregulation of Siah1 and the activation of the p38 MAPK/p53 pathway, decreased ethanol-induced apoptosis in NCCs and zebrafish embryos, and diminished ethanol-induced growth retardation and dysmorphology in zebrafish larvae. These results demonstrate that ethanol-induced downregulation of miR-135a contributes to ethanol-induced apoptosis in NCCs and developmental defects in zebrafish embryos by upregulating Siah1 and activating the p38 MAPK/p53 pathway.

\section{MATERIALS AND METHODS}

\section{Cell Culture and Ethanol Treatment}

Neural crest cells (JoMa1.3 cells) were cultured as described previously (Maurer et al., 2007; Chen et al., 2013a). Cells were grown on cell culture plates/dishes coated with fibronectin and maintained in Dulbecco's modified Eagle's medium (DMEM): Ham's F12 (1:1) at $37^{\circ} \mathrm{C}$ in $5 \% \mathrm{CO}_{2}$. NCCs were exposed to medium containing $50 \mathrm{mM}$ ethanol for $24 \mathrm{~h}$. The stable ethanol levels were maintained by using the methods described previously (Yan et al., 2010).

\section{Zebrafish Maintenance and Ethanol Treatment}

Adult $\mathrm{AB}$ zebrafish (Danio rerio) were obtained from the Zebrafish International Resource Center (ZIRC) at the University of Oregon, Eugene, OR, United States and maintained in $14 \mathrm{~h}: 10 \mathrm{~h}$ light: dark cycles at $28^{\circ} \mathrm{C}$. Fertilized eggs were collected after natural spawning and used for this study. For ethanol treatment, the zebrafish embryos at 3 hours post-fertilization (hpf) were treated with 0,1 , or $1.5 \%$ ethanol. At $24 \mathrm{hpf}$, the embryos were either collected for molecular analysis or transferred to fresh system water. These embryos transferred to fresh system water were collected at 4 or 5 days post-fertilization (dpf) for morphological analysis. This study was approved by the Institutional Animal Care and Use Committee of the University of Louisville. 


\section{Microinjection of miRNA Mimics Into Zebrafish Embryos}

Zebrafish embryos were microinjected at $1 \mathrm{hpf}$ with $2 \mathrm{nl}$ of synthetic miRNA-135a mimics and control mimics $(10 \mu \mathrm{M})$ prepared with $1 \times$ Danieau solution $[58 \mathrm{mM} \mathrm{NaCl}, 0.7 \mathrm{mM}$ $\mathrm{KCl}, 0.4 \mathrm{mM} \mathrm{MgSO}_{4}, 0.6 \mathrm{mM} \mathrm{Ca}\left(\mathrm{NO}_{3}\right)_{2}, 5.0 \mathrm{mM}$ HEPES, $\mathrm{pH}$ 7.6] by using a PLI-100A Plus Picoinjector (Harvard Apparatus, Holliston, MA, United States), as previously described (Pineda et al., 2005; Bill et al., 2009; Su et al., 2014). Successful injections were monitored via the co-injection with $0.1 \%$ Fast Green. After microinjection, embryos were transferred to a petri dish containing ethanol solution or system water for treatment from 3 to $24 \mathrm{hpf}$, as described above. Zebrafish embryos were collected at $24 \mathrm{hpf}$ for analysis of the expression of miR-135a and Siah1, and the analysis of apoptosis. Zebrafish larvae were collected at 4 and $5 \mathrm{dpf}$ for morphological analysis and craniofacial cartilage defect analysis, respectively.

\section{Analysis of miRNA Expression}

The expression of miRNA-135a in NCCs and zebrafish embryos was determined as previously described (Chen et al., 2015). Total RNA was isolated with mirVana miRNA Isolation Kit (Ambion, Austin, TX, United States), and quantitative RT-PCR was performed by using TaqMan MicroRNA assays (Ambion, Austin, TX, United States), following the manufacturer's instructions. All TaqMan microRNA assays were performed in triplicate. Data were normalized with snoRNA202 (NCCs) or U6 snRNA (zebrafish embryos) as endogenous controls. The relative expression of miR-135a was calculated using the comparative threshold cycle $(\mathrm{Ct})$ method as described previously (Chen et al., 2015).

\section{miRNA Mimics and Inhibitors Transfection}

For transient transfection, miRNA-135a mimics, miRNA inhibitors, control mimics, or control inhibitors at a final concentration of $50 \mathrm{nM}$ were transfected into NCCs using Lipofectamine 2000 (Life Technologies, Grand Island, NY, United States), following the manufacturer's instructions. The cells were harvested $48 \mathrm{~h}$ after transfection for additional treatments and analysis.

\section{Construction of Luciferase Reporter Plasmids and Reporter Assays}

MiRNA-135a target sites in the $3^{\prime}$-untranslated regions ( $3^{\prime}$-UTRs) of mouse Siahl mRNA were predicted by using Target Scan ${ }^{1}$ and microRNA ${ }^{2}$, and compared with the target sites reported in the previous study (Leung et al., 2014). The 3'-UTR of Siah1 containing putative miR-135a binding sites was amplified from mouse genomic DNA and cloned into the pMIR-REPORT ${ }^{\mathrm{TM}}$ (Ambion, Austin, TX, United States). Primers used to clone the DNA fragments containing the Siah1 $3^{\prime}$-UTR were: $5^{\prime}$ gactACTAGTtttcttttaactgacaagccatctgcgtggtcatagAAGCTTgcta

${ }^{1}$ http://www.targetscan.org/

${ }^{2}$ http://www.microrna.org/microrna/home/do $3^{\prime} ; \quad 5^{\prime} \quad$ tagcAAGCTTctatgaccacgcagatggcttgtcagttaaaagaaaACTAGTagtc $3^{\prime}$. The reporter assays were performed as previously described (Chen et al., 2015). In brief, NCCs were co-transfected with $200 \mathrm{ng}$ of constructed plasmids containing miR-135a binding sites, 20 ng Renilla luciferase pRL-TK control reporter vector (Promega, Madison, WI, United States) and $50 \mathrm{nM}$ of miRNA-135a mimics or mimic control (Ambion, Austin, TX, United States) using Lipofectamine 2000 (Invitrogen, Carlsbad, CA, United States) according to the manufacturer's protocol. Luciferase activity was then measured at $48 \mathrm{~h}$ after the transfection using the Dual-Luciferase assay kit (Promega, Madison, WI, United States) with a Lumat LB 9507 Ultra Sensitive Tube Luminometer (Berthold Technologies, Bad Wildbad, Germany). The luciferase activity of each sample was normalized to the $\mathrm{pRL} / \mathrm{TK}$-driven Renilla luciferase activity.

\section{Western Blotting}

Western blotting was performed by standard protocols as described previously (Chen et al., 2013b; Sun et al., 2014). Proteins were probed with the following antibodies: SIAH1 rabbit pAb (Abcam, Cambridge, MA, United States), $\beta$-Actin mouse mAb (Santa Cruz, Santa Cruz, CA, United States), Phosphop38 MAPK (Thr180/Tyr182) rabbit mAb (Cell Signaling Technology, Inc., Beverly, MA, United States), p38 MAPK rabbit pAb (Cell Signaling Technology, Inc., Beverly, MA, United States), Phospho-p53 (Ser15) rabbit pAb (Cell Signaling Technology, Inc., Beverly, MA, United States), p53 mouse mAb (Abcam, Cambridge, MA, United States), p53 upregulated modulator of apoptosis (PUMA) rabbit pAb (Abcam, Cambridge, MA, United States), Bcl-2 homologous antagonist/killer (Bak) rabbit pAb (Cell Signaling Technology, Inc., Beverly, MA, United States), and cleaved caspase-3 (Asp175) rabbit pAb (Cell Signaling Technology, Inc., Beverly, MA, United States). The membranes were developed on Molecular Imager ChemiDoc XRS + (Bio-Rad, Hercules, CA, United States), and the intensity of the protein band was analyzed by Image software $(1.48 \mathrm{~V}$, National Institutes of Health, United States). All Western blot analyses were performed in triplicate.

\section{Analysis of Apoptosis}

Apoptosis was determined by the analysis of caspase- 3 cleavage and activity, as well as the terminal deoxynucleotidyl transferase dUTP nick end labeling (TUNEL) assay. Caspase-3 cleavage was determined by Western blot as described previously (Dong et al., 2008; Chen et al., 2013a). Caspase-3 activity was determined by using Caspase-Glo ${ }^{\circledR}$ 3/7 Assay Systems (Promega, Madison, WI, United States). TUNEL assay was performed by using a TiterTACS In situ Detection Kit (Trevigen, Inc., Gaithersburg, MD, United States), following the manufacturer's protocol.

\section{Morphological Analysis and Whole-Mount Skeletal Staining}

Morphological analysis of zebrafish larvae at $4 \mathrm{dpf}$ was performed by using a stereoscopic microscope (Olympus SZX16, Tokyo, Japan). Whole-mount skeletal staining of zebrafish larvae at $5 \mathrm{dpf}$ was conducted with Alcian blue staining (Sigma Chemical, Co., 

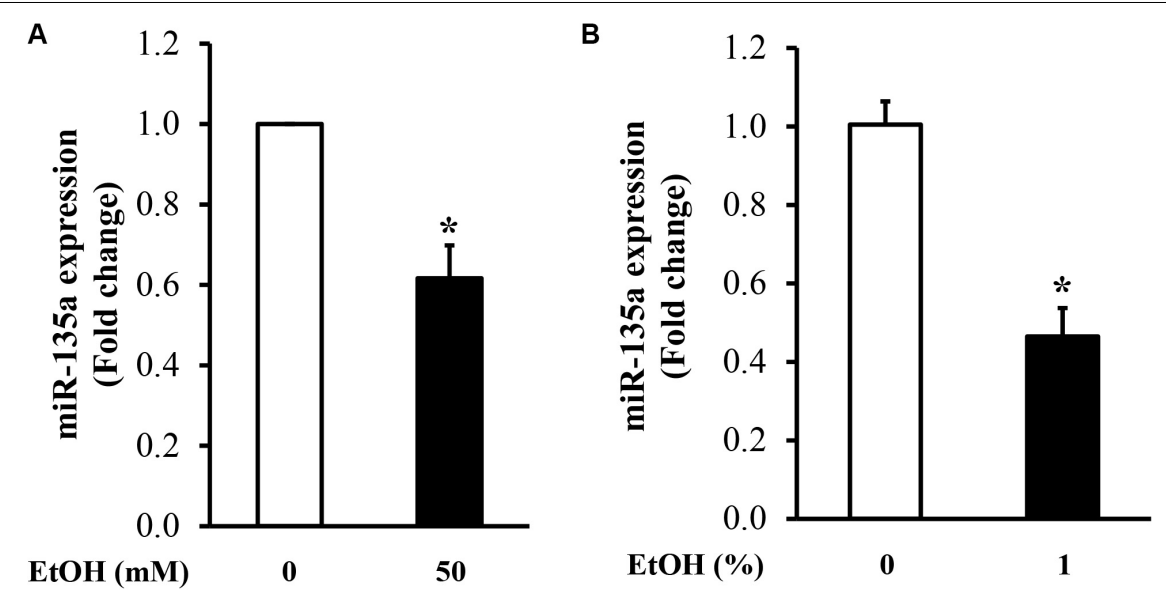

FIGURE 1 | Ethanol exposure decreased the expression of miR-135a in neural crest cells (NCCs) exposed to 50 mM ethanol for 24 h (A) and zebrafish embryos treated with $1 \%$ ethanol and collected at $24 \mathrm{~h}$ post-fertilization (hpf; B). The expression of miR-135a was determined by qRT-PCR, as described in the section "Materials and Methods". Data are expressed as fold change over control and represent the mean \pm SD of three separate experiments. ${ }^{\star} p<0.05$ vs. control.

St Louis, MO, United States), as described by others (Neuhauss et al., 1996; Walker and Kimmel, 2007), and was visualized by a stereoscopic microscope (Olympus SZX16, Tokyo, Japan) as previously described (Carvan et al., 2004).

\section{Statistical Analysis}

Statistical analyses were performed as described previously (Chen et al., 2013b) using GraphPad Prism software (GraphPad Software, San Diego, CA, United States). All data were expressed as means $\pm \mathrm{SD}$ of three separate experiments. One-way ANOVA was used to compare the difference between groups, and multiple comparison post-tests were conducted by using Bonferroni's test. Differences between groups were considered significant at $p<0.05$.

\section{RESULTS}

\section{Ethanol Treatment Significantly Decreased the Expression of miR-135a in NCCs and Zebrafish Embryos}

To determine the effect of ethanol treatment on the expression of miRNA-135a in NCCs, NCCs were exposed to $50 \mathrm{mM}$ ethanol for $24 \mathrm{~h}$. MiR-135a expression level was examined by qRT-PCR. As shown in Figure 1A, exposure of NCCs to $50 \mathrm{mM}$ ethanol for $24 \mathrm{~h}$ significantly decreased the expression of miR-135a in NCCs, indicating that ethanol treatment can downregulate the expression of miR-135a in NCCs. To determine whether ethanol exposure can also decrease the expression of miR-135a in vivo, zebrafish embryos at $3 \mathrm{hpf}$ were treated with or without $1 \%$ ethanol and collected at $24 \mathrm{hpf}$ for analysis of miR-135a expression using the qRT-PCR assay. As shown in Figure 1B, $1 \%$ ethanol treatment resulted in a significant reduction of miR135 a expression in zebrafish embryos. These results demonstrate that both in vitro and in vivo ethanol treatment can decrease the expression of miR-135a.

\section{Siah1 Is the Direct Target of miR-135a in NCCs}

Bioinformatics prediction indicated that Siah1 is a direct target of miR-135a (Figure 2A). Siah1 has also been validated to be a direct target of miRNA-135a in HeLa cells (Pang et al., 2011). To validate that Siah1 is also a direct target of miR-135a in NCCs, we cloned the $3^{\prime}$-UTR segments of Siah1 into the pMIR-Report vector to create a luciferase reporter system. NCCs were cotransfected with the vector containing pMIR reporter-luciferase fused with or without the $3^{\prime}$-UTR of Siah1 and miR135a mimic or miRNA control mimic. As shown in Figure 2B, co-transfected of the $3^{\prime}$-UTR of Siah1 mRNA and miR135a resulted in a significant reduction in luciferase activity as compared to the NCCs cotransfected with Siah1 $3^{\prime}$-UTR and control miRNA mimic. In addition, overexpression of miR-135a by transfecting with miR135a mimics greatly downregulated the protein expression of Siah1, while downregulation of endogenous miR-135a through transfecting with miR-135a inhibitors significantly elevated the protein expression of Siah1 (Figure 2C), demonstrating that Siah1 is a direct target of miR-135a in NCCs.

\section{Over-Expression of miR-135a Diminished Ethanol-Induced Upregulation of Siah1 in NCCs and Zebrafish Embryos}

To determine whether the downregulation of miR-135a contributes to ethanol-induced upregulation of Siah1, NCCs transfected with miR-135a mimics or control mimics were exposed to $50 \mathrm{mM}$ ethanol, and the protein expression of Siah1 was analyzed. As shown in Figure 3A, ethanol exposure resulted in a significant increase in the protein expression of Siah1. Overexpression of miR-135a by transfection with miR-135a mimics diminished ethanol-induced upregulation of Siah1 in NCCs, demonstrating that downregulation of miR-135a contributes to ethanol-induced upregulation of Siah1 in NCCs. To determine whether overexpression of miR-135a can also 
A

Siah1 5'......UUUCUUUUAACUGACAAGCCAUCUGCGUGGUCAUAG.....3
miR-135a $33^{\prime}$ AGUGUAUCCUUAUUUUUCGGUAU 5

B

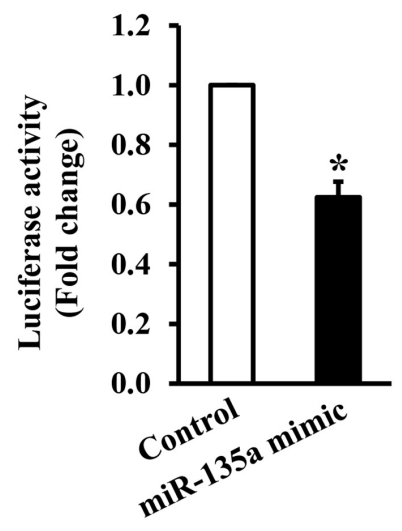

C
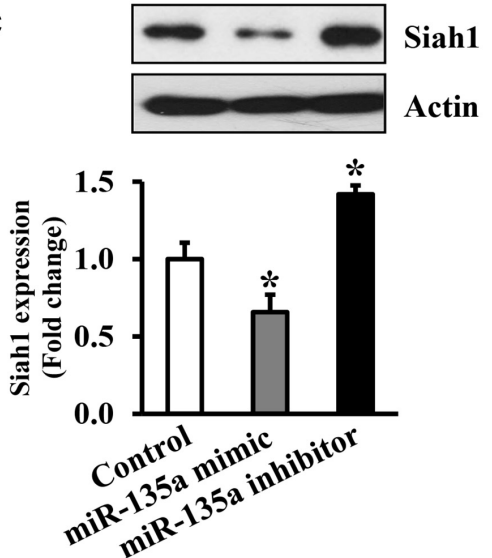

FIGURE 2 | Siah1 is the direct target of miR-135a in neural crest cells (NCCs). (A) The predicted binding sites of miR-135a in the 3'-UTR of Siah1 mRNA. (B) Luciferase reporter assays for the binding of miR-135a to the 3'-UTR of Siah1 in NCCs. (C) Western bolt analysis of the protein expression of Siah1 in NCCs transfected with miR-135a mimics or inhibitors for $48 \mathrm{~h}$. Data are expressed as fold change over control and represent the mean \pm SD of three separate experiments. ${ }^{*} p<0.05$ vs. control.

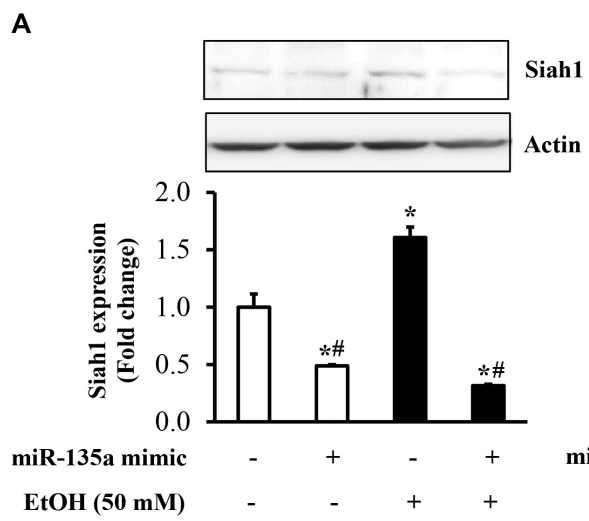

B
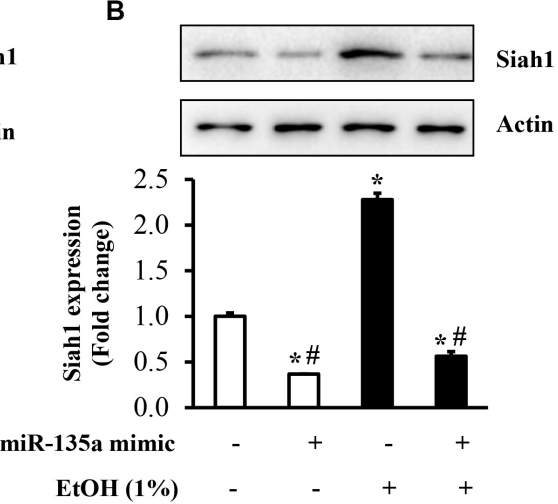

FIGURE 3 | Overexpression of miR-135a significantly decreased the ethanol-induced upregulation of Siah1 in neural crest cells (NCCs) and zebrafish embryos. NCCs transfected with control mimics or miR-135a mimics were treated with $50 \mathrm{mM}$ ethanol for $24 \mathrm{~h}$. Zebrafish embryos microinjected with control or miR-135a mimics were treated with $1 \%$ ethanol and collected at $24 \mathrm{~h}$ post-fertilization (hpf). The protein expression of Siah1 was examined in NCCs (A) and zebrafish embryos (B) by western blot. Data are expressed as fold change over control and represent the mean \pm SD of three separate experiments. ${ }^{*} p<0.05$ vs. control; \# $p<0.05$ vs. ethanol.

diminish ethanol-induced upregulation of Siah1 in zebrafish embryos, zebrafish embryos microinjected with miR-135a mimics and control mimics were exposed to $1 \%$ ethanol, and the protein expression of Siah1 was analyzed by western blot. As shown in Figure 3B, exposure to ethanol significantly increased the expression of Siahl. Microinjection of miR-135a mimics significantly diminished ethanol-induced upregulation of Siah1 in zebrafish embryos, indicating that overexpression of miR-135a can also prevent ethanol-induced upregulation of Siah1 in zebrafish embryos.

\section{Overexpression of miR-135a Diminished Ethanol-Induced Activation of the p38 MAPK/p53 Pathway in NCCs}

Our previous study has demonstrated that ethanol-induced upregulation of Siah1 triggered apoptosis in NCCs through promoting p38 MAPK-mediated activation of the p53 signaling pathway (Yuan et al., 2017). To determine whether the downregulation of miR-135a contributes to ethanol-induced apoptosis in NCCs through Siah1-mediated activation of the 


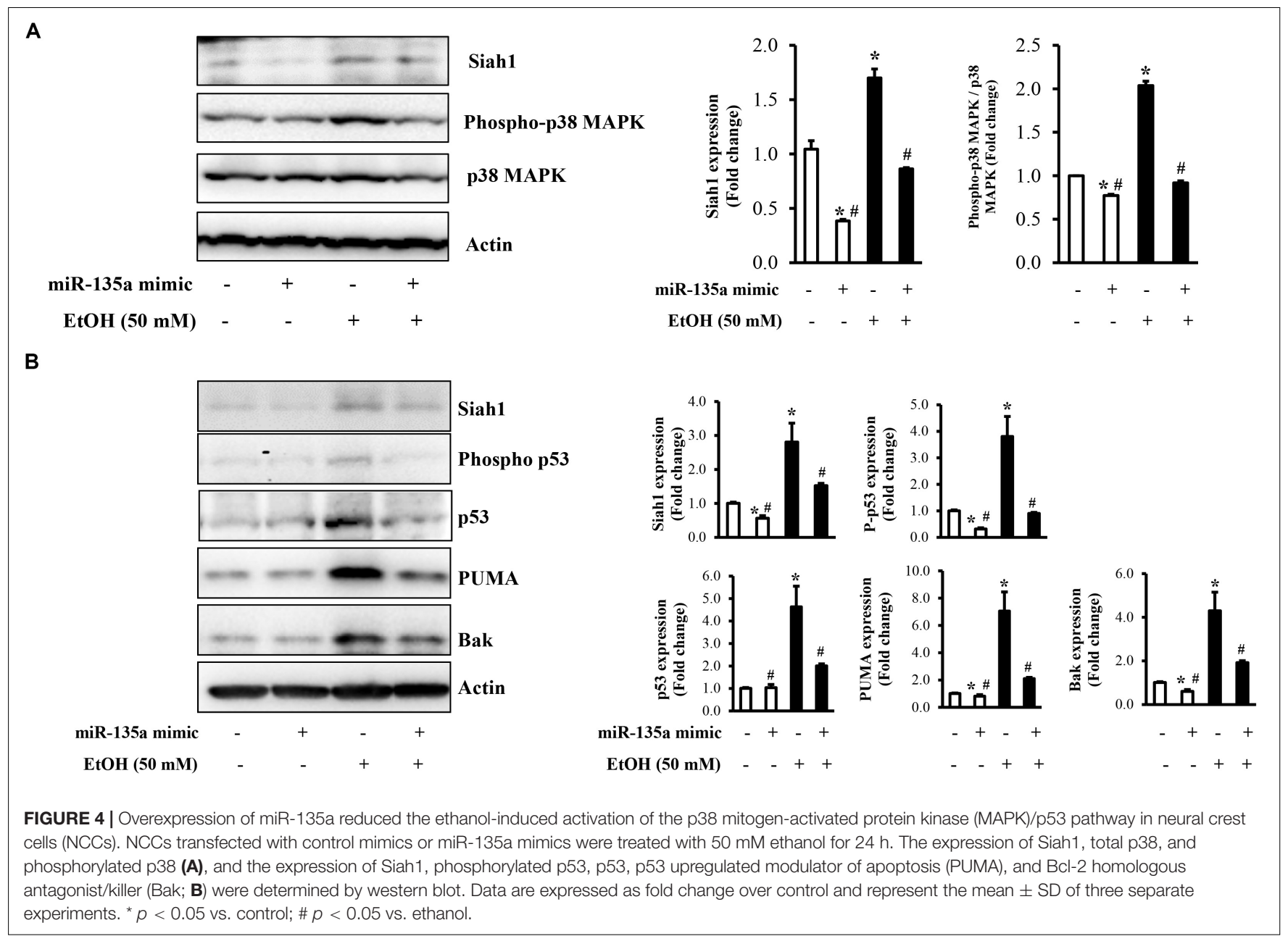

p38 MAPK/p53 pathway, we first examined whether the overexpression of miRNA-135a can inhibit the activation of the p38 MAPK pathway in ethanol-exposed NCCs. As shown in Figure 4A, ethanol treatment resulted in the activation of the p38 MAPK pathway, as indicated by enhanced phosphorylation of p38 MAPK, consisting with our previous studies (Yuan et al., 2017). Overexpression of miR-135a significantly diminished ethanol-induced expression of phosphor-p38 MAPK, indicating that miR-135a can inhibit the p38 MAPK pathway through downregulating Siah1. To determine the role of miRNA-135a on ethanol-induced apoptosis in NCCs, we next examined the p53 apoptotic pathway, which is the downstream targets of the p38 MAPK pathway that has been shown to be regulated tightly by Siah1 in NCCs (Yuan et al., 2017). We found that ethanol exposure increased the total protein levels of p53 and the levels of the phosphorylated p53. Ethanol treatment also significantly increased the expression of p53 downstream targets, PUMA and Bak. Overexpression of miRNA-135a significantly diminished ethanol-induced increases in the phosphorylation and total protein level of p53, and the expression of PUMA and Bak (Figure 4B), indicating that miRNA-135a can modulate the p38 MAPK/p53 apoptotic pathway in NCCs through targeting Siah1.

\section{Overexpression of miR-135a} Significantly Reduced Ethanol-Induced Activation of Caspase-3 and Diminished Ethanol-Induced Apoptosis in NCCs and Zebrafish Embryos

To determine whether overexpression of miR-135a can prevent ethanol-induced apoptosis in NCCs, NCCs transfected with miR-135a mimics were exposed to $50 \mathrm{mM}$ ethanol for $24 \mathrm{~h}$. As shown in Figures 5A,B, ethanol exposure resulted in a significant increase in caspase- 3 cleavage and activity in NCCs, indicating that ethanol exposure can increase apoptosis in NCCs that was confirmed by the TUNEL assay (Figure 5C). Overexpression of miR-135a significantly diminished ethanolinduced increases in caspase- 3 cleavage and activity and reduced apoptosis in ethanol-exposed NCCs (Figures 5A-C). These results demonstrate that overexpression of miR-135a can prevent ethanol-induced apoptosis in NCCs. To determine whether the upregulation of miR-135a can also prevent ethanol-induced apoptosis in zebrafish embryos, zebrafish embryos microinjected with miR-135a mimics and control mimics were exposed to ethanol from 3 to $24 \mathrm{hpf}$. As shown in Figure 5D, microinjection of miR-135a mimics significantly reduced ethanol-induced 
A

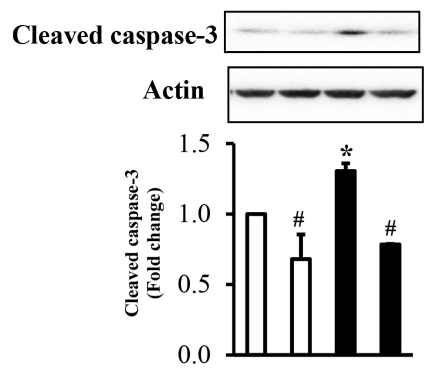

miR-135a mimic EtOH (50 mM) - - + +

C

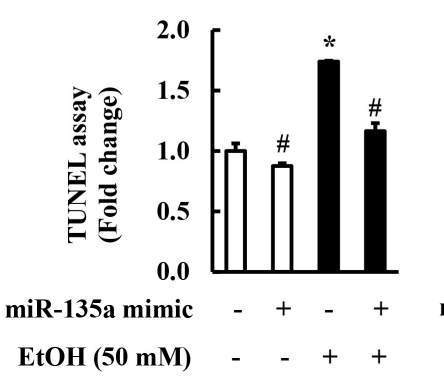

B

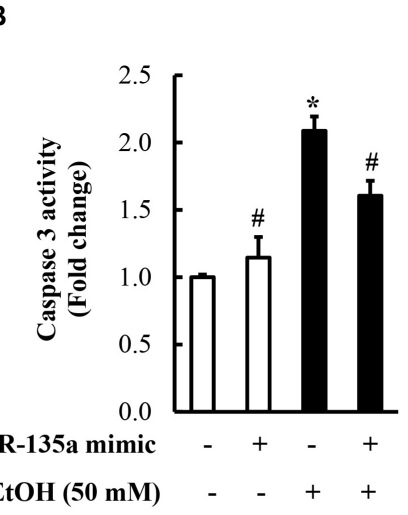

D

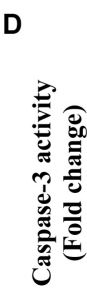

miR-135a mimic



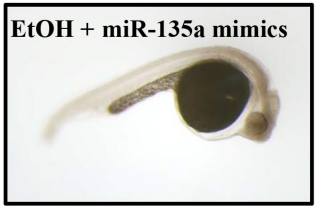

E
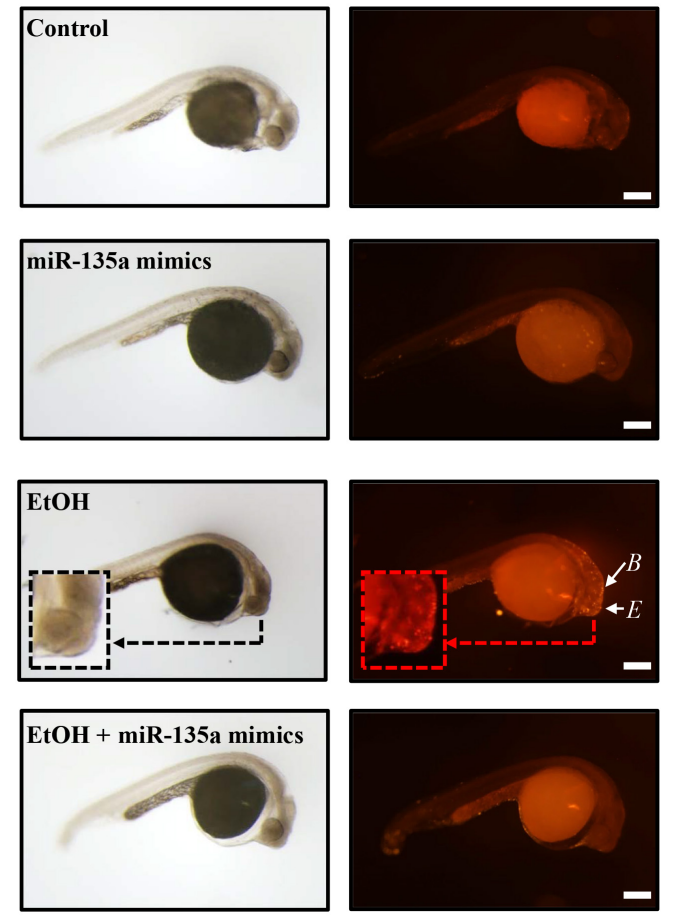

FIGURE 5 | Overexpression of miR-135a significantly decreased ethanol-induced caspase-3 activation and apoptosis in neural crest cells (NCCs) and zebrafish embryos. NCCs transfected with control mimics or miR-135a mimics were treated with $50 \mathrm{mM}$ ethanol for $24 \mathrm{~h}$. Zebrafish embryos microinjected with control or miR-135a mimics were treated with 1\% ethanol and collected at $24 \mathrm{~h}$ post-fertilization (hpf). Ethanol-induced apoptosis in NCCs (A-C) and zebrafish embryos (D,E) was determined by analysis of caspase-3 cleavage (A), caspase-3 activity (B,D), and terminal deoxynucleotidyl transferase dUTP nick end labeling (TUNEL) analysis $(\mathbf{C}, \mathbf{E})$. White arrows in $\mathbf{( E )}$ indicate brain $\mathbf{( B )}$ and eye $\mathbf{( E )}$ in zebrafish embryos. Data are expressed as fold change over control and represent the mean \pm SD of three separate experiments. ${ }^{*} p<0.05$ vs. control; $\# p<0.05$ vs. ethanol. Scale bar $=200 \mu \mathrm{m}$.

increase in caspase-3 activity in zebrafish embryos. Whole-mount TUNEL staining also shown that ethanol-induced apoptosis was significantly attenuated by the microinjection of miR135 a mimics in zebrafish embryos, especially in the brain and eye (Figure 5E).

\section{Microinjection of miR-135a Mimics Attenuated Ethanol-Induced Growth Retardation and Dysmorphology in Zebrafish Larvae}

It has been reported that ethanol-induced apoptosis in NCCs contributes heavily to the subsequent abnormalities that are characteristics of FASD (Kotch and Sulik, 1992a; Cartwright and Smith, 1995a). To determine whether microinjection of miR-135a mimics can attenuate ethanol-induced growth retardation and dysmorphology in zebrafish, zebrafish embryos microinjected with control or miR-135a mimics were treated with $1.5 \%$ ethanol from 3 to $24 \mathrm{hpf}$ and then were allowed to grow in system water without ethanol. Zebrafish larvae from control and treated groups were collected at $4 \mathrm{dpf}$ for morphological analysis. As shown in Figure 6, ethanol treatment resulted in significant growth retardation in zebrafish larvae, as indicated by the dramatically reduced body length. Ethanol exposure also resulted in significant developmental defects, including small eyes, microcephaly, and pericardial edema. Microinjection of miR-135a mimics significantly attenuated the ethanol-induced growth retardation and dysmorphology in zebrafish larvae.

\section{Microinjection of miR-135a Mimics Significantly Diminished Ethanol-Induced Craniofacial Cartilage Defects in Zebrafish Larvae}

To determine whether the microinjection of miR-135a mimics can attenuate the ethanol-induced craniofacial cartilage defects in zebrafish larvae, zebrafish embryos microinjected with control or miRNA-135a mimics were treated with $1.5 \%$ ethanol from 3 to $24 \mathrm{hpf}$. Zebrafish larvae were collected at $5 \mathrm{dpf}$ and stained with Alcian blue. As shown in Figure 7A, ethanol exposure resulted in an overall reduction in head size and micrognathia due to reduced jaw outgrowth in zebrafish larvae. A closer examination of craniofacial cartilage at $5 \mathrm{dpf}$ larvae revealed that the length of the mandibular arch cartilages (lower jaw), including the ventral Meckel's cartilage (m) and dorsal palatoquadrate (pq), were significantly reduced in ethanolexposed zebrafish larvae, as compared to control. In addition, exposure to ethanol at the embryonic stage significantly reduced 




FIGURE 6 | Microinjection of miR-135a mimics diminished ethanol-induced growth retardation and dysmorphology in zebrafish larvae. Embryos microinjected with control or miR-135a mimics were treated with 1.5\% ethanol from 3 to $24 \mathrm{~h}$ post-fertilization (hpf). Zebrafish larvae were collected at 4 days post-fertilization (dpf) for morphological analysis. Analysis of ethanol-induced growth retardation and dysmorphology was performed by using a stereoscopic microscope. The black arrows indicate the heart and the arrowheads indicate eye. Data represent the mean $\pm \mathrm{SD}$ of three separate experiments. ${ }^{*} p<0.05$ vs. control; \# $p<0.05$ vs. ethanol.

the length of hyosymplectic (hs) and ceratohyal (ch), and the distance between $\mathrm{m}$ and $\mathrm{pq}$ joint and between the arch of $\mathrm{m}$ and basihyal (bh; Figure 7B). Embryonic ethanol treatment also led to the abnormal angulation of the ch cartilage (Figure 7C). Overexpression of miR-135a significantly diminished ethanolinduced reduction in the length of $\mathrm{m}, \mathrm{pq}$, hs, and ch and the distance between $\mathrm{m}$ and $\mathrm{pq}$ joints and between $\mathrm{m}$ and bh. Microinjection of miR-135a also attenuated the ethanolinduced abnormal angulation of ch cartilage, indicating that the upregulation of miR-135a can attenuate ethanol-induced craniofacial cartilage defects in zebrafish larvae (Figures 7A-C).

\section{DISCUSSION}

Growing evidence suggests that impairment of differentiation, migration, and survival of NCCs is a major component of the pathogenesis of FASD and that dysregulation of gene expression is a key driver of the ethanol-induced impairment in NCCs (Kotch and Sulik, 1992b; Cartwright and Smith, 1995b; Rovasio and Battiato, 2002; Steventon et al., 2014; Zhang et al., 2017; Yuan et al., 2018; Fan et al., 2019; Li et al., 2019). MicroRNAs have been shown to be involved in the ethanol-induced impairment of NCCs. Our previous studies have shown that miR-125b can protect against ethanol-induced apoptosis in NCCs and mouse embryos by targeting Bak1 and PUMA and that microinjection of miR-125b mimics can prevent ethanol-induced embryotoxicity (Chen et al., 2015). We have also demonstrated that miR-34a can mediate ethanol-induced impairment of neural differentiation of
NCCs by targeting autophagy-related gene 9a (Fan et al., 2019). In the present study, we found that ethanol exposure significantly reduced the expression of miR-135a, increased apoptosis in NCCs exposed to $50 \mathrm{mM}$ ethanol and zebrafish embryos exposed to $1.0 \%$ ethanol, and resulted in growth retardation and developmental defects in zebrafish larvae exposed to $1.5 \%$ ethanol. There is a consensus that the ethanol levels in the zebrafish embryo tissue are approximately 25-35\% of the levels of the medium (Flentke et al., 2014; Lovely et al., 2014). Therefore, 1.0 or $1.5 \%$ ethanol in the medium is $\sim 300$ or $450 \mathrm{mg} / \mathrm{dL}$ in the embryo tissue. These ethanol concentrations were chosen because studies have shown that a peak maternal blood alcohol concentration of $400-500 \mathrm{mg} / \mathrm{dL}$ (approximately $85-105 \mathrm{mM}$ ) is needed to produce major malformations with the characteristics of fetal alcohol syndrome in mouse embryos (Sulik et al., 1981; Kotch and Sulik, 1992a; Dunty et al., 2001). These ethanol concentrations are relatively high but are not beyond that which can be observed in chronic alcoholics (Adachi et al., 1991).

MiR-135a is a member of the miR-135 superfamily. A number of studies have reported that the downregulation of miR135a was involved in tumorigenesis in a variety of cancers, in which miR-135a exerts tumor-suppressive effects (Yamada et al., 2013; Kroiss et al., 2015; Zhao et al., 2017). In contrast, other studies have shown that miR-135a is an oncogenic miRNA in colorectal carcinomas (Nagel et al., 2008) and that overexpression of miR-135a promoted tumorigenesis of portal vein tumor thrombus (Liu et al., 2012) and enhanced the growth of HeLa- and NC104-E6/E7-derived tumor (Leung et al., 2014). Studies have also shown that miR-135a is involved in 
A
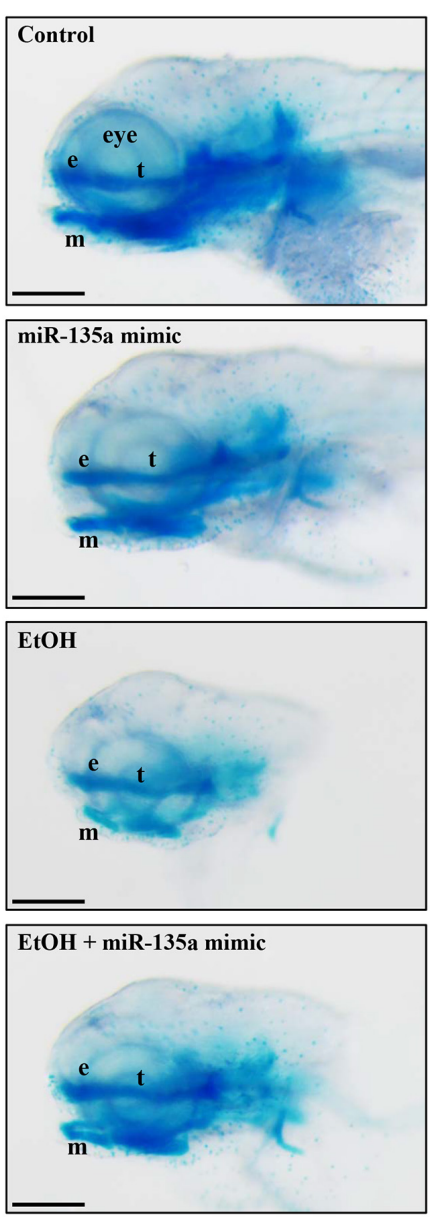
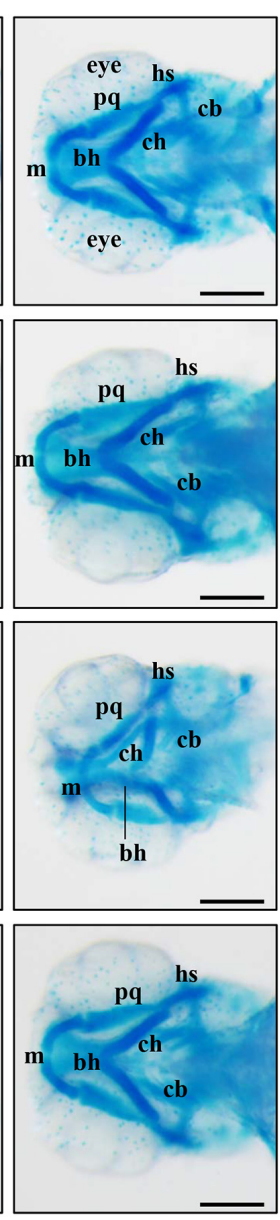

B


Distance between

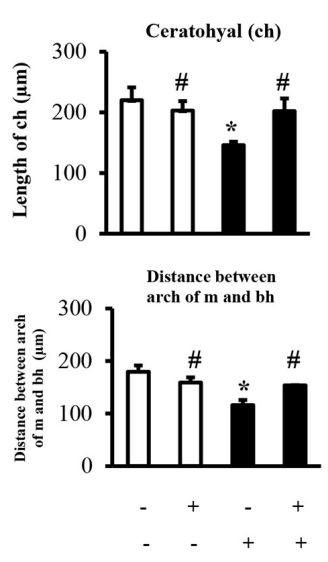

C


EtOH

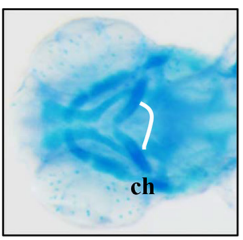

EtOH+ miR-135a mimics



FIGURE 7 | Microinjection of miR-135a mimics attenuated ethanol-induced craniofacial cartilage defects in zebrafish larvae. Embryos microinjected with control or miR-135a mimics were treated with 1.5\% ethanol from $3 \mathrm{~h}$ post-fertilization (hpf) to 24 hpf. Zebrafish larvae were collected at 5 days post-fertilization (dpf) for skeletal staining with Alcian blue. (A) Lateral and ventral view of Alcian blue-stained craniofacial cartilages in larvae from different treatment groups. (B) Morphometric analysis of the craniofacial cartilages in larvae from different treatment groups. The length of Meckel's cartilage (m), palatoquadrate (pq), hyosymplectic (hs), ceratohyal (ch), and the distance between $\mathrm{m}$ and pq joint, and between the arch of $\mathrm{m}$ and basihyal (bh) were measured. (C) Comparisons of ceratohyal cartilage (ch) angle between different treatment groups. Data represent the mean $\pm \mathrm{SD}$ of three separate experiments. ${ }^{*} p<0.05$ vs. control; \# $p<0.05$ vs. ethanol. e, ethmoid plate; t, trabeculae cranii; cb, ceratobranchial; bh, basihyal. Scale bar $=200 \mu \mathrm{m}$.

brain inflammation and angiogenesis in Alzheimer's disease (Ko et al., 2015). In addition, it has been reported that miR-135a can promote proliferation and prevent apoptosis. For example, a study by Dong et al., indicated that, by targeting HIF-1 $\alpha$, miR-135a downregulated pro-apoptotic genes $\mathrm{Bax}$ and $\mathrm{Bad}$, upregulated anti-apoptotic gene $\mathrm{Bcl}-2$, resulting in increased proliferation and reduced apoptosis of astrocytes in the bacterial meningitis rat models (Dong et al., 2019). Upregulation of miR-135a also protected human umbilical vein endothelial cells against mechanical stretch-induced increases in apoptosis and ventilator-induced lung injury through activating PI3K/Akt signaling pathway by targeting $\mathrm{PH}$ domain leucine-rich repeat-containing protein phosphatase 2 (PHLPP2) (Yan et al., 2018). In the present study, we have shown that ethanol-induced downregulation of miR-135a resulted in the upregulation of Siah1 and that overexpression of miR135a significantly reduced ethanol-induced upregulation of
Siah1, indicating that downregulation of miR-135a contributes to ethanol-induced upregulation of Siah1 in NCCs and zebrafish embryos.

Siah is a member of a highly conserved family of E3 ubiquitin ligases (Carthew and Rubin, 1990). Siah ligases are involved in the ubiquitination and proteasomal degradation of several proteins that are essential for a variety of signaling pathways, including membrane receptors (Liani et al., 2004; Winter et al., 2008), a microtubule-associated motor protein (Linares-Cruz et al., 1998) and transcriptional regulators (Zhang et al., 1998; Tiedt et al., 2001). The upregulation of Siah1 has also been found to induce cell-cycle arrest and the induction of apoptosis (Relaix et al., 2000; Matsuzawa and Reed, 2001). We have also demonstrated that ethanol treatment can significantly increase the expression and nuclear translocation of Siah1 in NCCs and that Siah1 signaling plays a critical role in ethanol-induced apoptosis in NCCs (Sun et al., 2014). In addition, we have shown that 
ethanol-induced up-regulation of Siah1 can induce apoptosis in NCCs through p38 MAPK-mediated activation of the p53 signaling pathway (Yuan et al., 2017). In this study, we found that, in addition to NCCs, ethanol exposure can also increase the expression of Siah1 in zebrafish embryos. We also found that ethanol-induced upregulation of Siah1 resulted in the activation of p38 MAPK/p53 pathway and that overexpression of miR135 a significantly diminished ethanol-induced activation of p38 MAPK/p53 pathway. These results consist of the findings from our previous study and demonstrate that the upregulation of miR-135a can inhibit p38 MAPK-mediated activation of the p53 signaling pathway through downregulating Siah1.

It is well known that p38 MAPK pathway can modulate apoptosis by regulating p53 pathway (Zheng et al., 2005; Gao et al., 2014) and that $\mathrm{p} 53$ is a transcription factor that regulates the expression of genes involved in apoptosis (Kho et al., 2004; Schuler and Green, 2005). Previous studies from our laboratory have shown that knockdown of Siah1 by siRNA significantly diminished the ethanol-induced increase in the phosphorylation of p38 MAPK, and significantly decreased ethanol-induced increases in p53 stability and its phosphorylation in NCCs, and that p38 MAPK activation is essential for ethanol-induced Siah1-mediated p53 activation, leading to apoptosis in ethanolexposed NCCs (Yuan et al., 2017). In this study, we found that ethanol treatment significantly increased the expression of p53 downstream targets, PUMA and Bak, the activation of caspase3 and apoptosis in NCCs. Overexpression of miRNA-135a significantly diminished ethanol-induced upregulation of PUMA and Bak and apoptosis in NCCs. In addition, microinjection of miR-135a mimics dramatically reduced the apoptosis in zebrafish embryos and diminished ethanol-induced growth retardation and dysmorphology in zebrafish larvae. These results demonstrate that the upregulation of miR-135a can prevent ethanol-induced apoptosis in NCCs and developmental defects in zebrafish embryos by modulating the Siah1-mediated p38 $\mathrm{MAPK} / \mathrm{p} 53$ pathway.

\section{CONCLUSION}

The present study has demonstrated that ethanol treatment decreased the expression of miR-135a and thereby increased the expression of its direct target, Siah1, which, in turn, activated the p38 MAPK/p53 pathway, increased apoptosis

\section{REFERENCES}

Adachi, J., Mizoi, Y., Fukunaga, T., Ogawa, Y., Ueno, Y., and Imamichi, H. (1991). Degrees of alcohol intoxication in 117 hospitalized cases. J. Stud. Alcohol 52, 448-453. doi: 10.15288/jsa.1991.52.448

Beis, D., and Stainier, D. Y. (2006). In vivo cell biology: following the zebrafish trend. Trends Cell Biol. 16, 105-112. doi: 10.1016/j.tcb.2005.12.001

Bill, B. R., Petzold, A. M., Clark, K. J., Schimmenti, L. A., and Ekker, S. C. (2009). A primer for morpholino use in zebrafish. Zebrafish 6, 69-77. doi: 10.1089/zeb. 2008.0555

Bilotta, J., Barnett, J. A., Hancock, L., and Saszik, S. (2004). Ethanol exposure alters zebrafish development: a novel model of fetal alcohol syndrome. Neurotoxicol. Teratol. 26, 737-743. doi: 10.1016/j.ntt.2004.06.011 in NCCs and zebrafish embryos and resulted in growth retardation and developmental defects. Overexpression of miR135a significantly reduced ethanol-induced upregulation of Siah1 and the activation of the p38 MAPK/p53 pathway, decreased ethanol-induced apoptosis in NCCs and zebrafish embryos, and diminished ethanol-induced growth retardation and dysmorphology in zebrafish larvae. These results demonstrate that ethanol-induced downregulation of miR-135a contributes to ethanol-induced apoptosis in NCCs and craniofacical defects in zebrafish embryos by upregulating Siah1 and activating the p38 MAPK/p53 pathway. These findings elucidate the mechanisms by which miR-135a modulates ethanol-induced apoptosis in NCCs and craniofacial defects in zebrafish embryos and suggest that miR-135a may represent a novel therapeutic target for the intervention and prevention of FASD.

\section{DATA AVAILABILITY STATEMENT}

All datasets generated in this study are included in the article.

\section{ETHICS STATEMENT}

The animal study was reviewed and approved by Institutional Animal Care and Use Committee of the University of Louisville.

\section{AUTHOR CONTRIBUTIONS}

FY and SC conceptualized and designed the experiments and participated in data interpretation and manuscript preparation. FY, YY, and JL performed the experiments and participated in data analysis. HF, YL, LL, and WF participated in data interpretation and discussion. All authors reviewed the manuscript.

\section{FUNDING}

This work was supported by the National Institutes of Health Grants AA020265, AA028435, AA021434, AA024337 (SC), and AA023190 (WF) from the National Institute on Alcohol Abuse and Alcoholism.

Carthew, R. W., and Rubin, G. M. (1990). Seven in absentia, a gene required for specification of R7 cell fate in the Drosophila eye. Cell 63, 561-577. doi: 10.1016/0092-8674(90)90452-k

Cartwright, M. M., and Smith, S. M. (1995a). Increased cell death and reduced neural crest cell numbers in ethanol-exposed embryos: partial basis for the fetal alcohol syndrome phenotype. Alcohol Clin. Exp. Res. 19, 378-386.

Cartwright, M. M., and Smith, S. M. (1995b). Stage-dependent effects of ethanol on cranial neural crest cell development: partial basis for the phenotypic variations observed in fetal alcohol syndrome. Alcohol Clin. Exp. Res. 19, 1454-1462. doi: 10.1111/j.1530-0277.1995.tb01007.x

Carvan, M. J. III, Loucks, E., Weber, D. N., and Williams, F. E. (2004). Ethanol effects on the developing zebrafish: neurobehavior and skeletal morphogenesis. Neurotoxicol. Teratol. 26, 757-768. doi: 10.1016/j.ntt.2004.06.016 
Chen, X., Liu, J., and Chen, S. Y. (2013a). Over-expression of Nrf2 diminishes ethanol-induced oxidative stress and apoptosis in neural crest cells by inducing an antioxidant response. Reprod. Toxicol. 42, 102-109. doi: 10.1016/j.reprotox. 2013.08.003

Chen, X., Liu, J., and Chen, S. Y. (2013b). Sulforaphane protects against ethanolinduced oxidative stress and apoptosis in neural crest cells by the induction of Nrf2-mediated antioxidant response. Br. J. Pharmacol. 169, 437-448. doi: 10.1111/bph.12133

Chen, X., Liu, J., Feng, W. K., Wu, X., and Chen, S. Y. (2015). MiR-125b protects against ethanol-induced apoptosis in neural crest cells and mouse embryos by targeting Bak 1 and PUMA. Exp. Neurol. 271, 104-111. doi: 10.1016/j. expneurol.2015.04.026

Dash, S., and Trainor, P. A. (2020). The development, patterning and evolution of neural crest cell differentiation into cartilage and bone. Bone 137:115409. doi: 10.1016/j.bone.2020.115409

Della, N. G., Senior, P. V., and Bowtell, D. D. (1993). Isolation and characterisation of murine homologues of the Drosophila seven in absentia gene (sina). Development 117, 1333-1343.

Dong, J., Sulik, K. K., and Chen, S. Y. (2008). Nrf2-mediated transcriptional induction of antioxidant response in mouse embryos exposed to ethanol in vivo: implications for the prevention of fetal alcohol spectrum disorders. Antioxid. Redox Signal. 10, 2023-2033. doi: 10.1089/ars.2007.2019

Dong, Y., Wang, J., Du, K. X., Jia, T. M., Zhu, C. L., Zhang, Y., et al. (2019). MicroRNA-135a participates in the development of astrocytes derived from bacterial meningitis by downregulating HIF-1alpha. Am. J. Physiol. Cell Physiol. 316, C711-C721. doi: 10.1152/ajpcell.00440.2018

Dunty, W. C. Jr., Chen, S. Y., Zucker, R. M., Dehart, D. B., and Sulik, K. K. (2001). Selective vulnerability of embryonic cell populations to ethanol-induced apoptosis: implications for alcohol-related birth defects and neurodevelopmental disorder. Alcohol Clin. Exp. Res. 25, 1523-1535.

Fan, H., Yuan, F., Yun, Y., Wu, T., Lu, L., Liu, J., et al. (2019). MicroRNA34a mediates ethanol-induced impairment of neural differentiation of neural crest cells by targeting autophagy-related gene 9a. Exp. Neurol. 320:112981. doi: 10.1016/j.expneurol.2019.112981

Fernandes, Y., and Gerlai, R. (2009). Long-term behavioral changes in response to early developmental exposure to ethanol in zebrafish. Alcohol Clin. Exp. Res. 33, 601-609. doi: 10.1111/j.1530-0277.2008.00874.x

Flentke, G. R., Klingler, R. H., Tanguay, R. L., Carvan, M. J. III, and Smith, S. M. (2014). An evolutionarily conserved mechanism of calcium-dependent neurotoxicity in a zebrafish model of fetal alcohol spectrum disorders. Alcohol Clin. Exp. Res. 38, 1255-1265. doi: 10.1111/acer.12360

Gao, J., Gao, J., Qian, L., Wang, X., Wu, M., Zhang, Y., et al. (2014). Activation of p38-MAPK by CXCL4/CXCR3 axis contributes to p53-dependent intestinal apoptosis initiated by 5-fluorouracil. Cancer Biol. Ther. 15, 982-991. doi: 10 . 4161/cbt.29114

Hong, F., Kim, W. H., Tian, Z., Jaruga, B., Ishac, E., Shen, X., et al. (2002). Elevated interleukin-6 during ethanol consumption acts as a potential endogenous protective cytokine against ethanol-induced apoptosis in the liver: involvement of induction of Bcl-2 and $\mathrm{Bcl}-\mathrm{x}(\mathrm{L})$ proteins. Oncogene 21, 32-43. doi: 10.1038/ sj.onc. 1205016

Jana, K., Jana, N., De, D. K., and Guha, S. K. (2010). Ethanol induces mouse spermatogenic cell apoptosis in vivo through over-expression of Fas/Fas-L, p53, and caspase- 3 along with cytochrome $\mathrm{c}$ translocation and glutathione depletion. Mol. Reprod. Dev. 77, 820-833. doi: 10.1002/mrd.21227

Kho, P. S., Wang, Z., Zhuang, L., Li, Y., Chew, J. L., Ng, H. H., et al. (2004). p53regulated transcriptional program associated with genotoxic stress-induced apoptosis. J. Biol. Chem. 279, 21183-21192. doi: 10.1074/jbc.M311912200

Ko, C. Y., Chu, Y. Y., Narumiya, S., Chi, J. Y., Furuyashiki, T., Aoki, T., et al. (2015). CCAAT/enhancer-binding protein delta/miR135a/thrombospondin 1 axis mediates PGE2-induced angiogenesis in Alzheimer's disease. Neurobiol. Aging 36, 1356-1368. doi: 10.1016/j.neurobiolaging.2014.11.020

Kotch, L. E., and Sulik, K. K. (1992a). Experimental fetal alcohol syndrome: proposed pathogenic basis for a variety of associated facial and brain anomalies. Am. J. Med. Genet. 44, 168-176. doi: 10.1002/ajmg.1320440210

Kotch, L. E., and Sulik, K. K. (1992b). Patterns of ethanol-induced cell death in the developing nervous system of mice; neural fold states through the time of anterior neural tube closure. Int. J. Dev. Neurosci. 10, 273-279. doi: 10.1016/ 0736-5748(92)90016-s
Kroiss, A., Vincent, S., Decaussin-Petrucci, M., Meugnier, E., Viallet, J., Ruffion, A., et al. (2015). Androgen-regulated microRNA-135a decreases prostate cancer cell migration and invasion through downregulating ROCK1 and ROCK2. Oncogene 34, 2846-2855. doi: 10.1038/onc.2014.222

Leung, C. O., Deng, W., Ye, T. M., Ngan, H. Y., Tsao, S. W., Cheung, A. N., et al. (2014). miR-135a leads to cervical cancer cell transformation through regulation of beta-catenin via a SIAH1-dependent ubiquitin proteosomal pathway. Carcinogenesis 35, 1931-1940. doi: 10.1093/carcin/bgu032

Li, Y., Yuan, F., Wu, T., Lu, L., Liu, J., Feng, W., et al. (2019). Sulforaphane protects against ethanol-induced apoptosis in neural crest cells through restoring epithelial-mesenchymal transition by epigenetically modulating the expression of Snail1. Biochim. Biophys. Acta Mol. Basis Dis. 1865, 2586-2594. doi: 10.1016/ j.bbadis.2019.07.002

Liani, E., Eyal, A., Avraham, E., Shemer, R., Szargel, R., Berg, D., et al. (2004). Ubiquitylation of synphilin-1 and alpha-synuclein by SIAH and its presence in cellular inclusions and Lewy bodies imply a role in Parkinson's disease. Proc. Natl. Acad. Sci. U.S.A. 101, 5500-5505. doi: 10.1073/pnas.0401081101

Linares-Cruz, G., Bruzzoni-Giovanelli, H., Alvaro, V., Roperch, J. P., Tuynder, M., Schoevaert, D., et al. (1998). p21WAF-1 reorganizes the nucleus in tumor suppression. Proc. Natl. Acad. Sci. U.S.A. 95, 1131-1135. doi: 10.1073/pnas.95. 3.1131

Liu, S., Guo, W., Shi, J., Li, N., Yu, X., Xue, J., et al. (2012). MicroRNA-135a contributes to the development of portal vein tumor thrombus by promoting metastasis in hepatocellular carcinoma. J. Hepatol. 56, 389-396. doi: 10.1016/j. jhep.2011.08.008

Lovely, C. B., Nobles, R. D., and Eberhart, J. K. (2014). Developmental age strengthens barriers to ethanol accumulation in zebrafish. Alcohol 48, 595-602. doi: 10.1016/j.alcohol.2014.06.003

Mantha, K., Laufer, B. I., and Singh, S. M. (2014). Molecular changes during neurodevelopment following second-trimester binge ethanol exposure in a mouse model of fetal alcohol spectrum disorder: from immediate effects to long-term adaptation. Dev. Neurosci. 36, 29-43. doi: 10.1159/000357496

Marrs, J. A., Clendenon, S. G., Ratcliffe, D. R., Fielding, S. M., Liu, Q., and Bosron, W. F. (2010). Zebrafish fetal alcohol syndrome model: effects of ethanol are rescued by retinoic acid supplement. Alcohol 44, 707-715. doi: 10.1016/j. alcohol.2009.03.004

Matsuzawa, S. I., and Reed, J. C. (2001). Siah-1, SIP, and Ebi collaborate in a novel pathway for beta-catenin degradation linked to p53 responses. Mol. Cell 7, 915-926. doi: 10.1016/s1097-2765(01)00242-8

Maurer, J., Fuchs, S., Jager, R., Kurz, B., Sommer, L., and Schorle, H. (2007). Establishment and controlled differentiation of neural crest stem cell lines using conditional transgenesis. Differentiation 75, 580-591. doi: 10.1111/j.1432-0436. 2007.00164.x

McCarthy, N., Wetherill, L., Lovely, C. B., Swartz, M. E., Foroud, T. M., and Eberhart, J. K. (2013). Pdgfra protects against ethanol-induced craniofacial defects in a zebrafish model of FASD. Development 140, 3254-3265. doi: 10. 1242/dev.094938

Mukherjee, R. A., Hollins, S., and Turk, J. (2006). Fetal alcohol spectrum disorder: an overview. J. R. Soc. Med. 99, 298-302. doi: 10.1258/jrsm.99.6.298

Muralidharan, P., Sarmah, S., Zhou, F. C., and Marrs, J. A. (2013). Fetal alcohol spectrum disorder (FASD) associated neural defects: complex mechanisms and potential therapeutic targets. Brain Sci. 3, 964-991. doi: 10.3390/ brainsci3020964

Nagel, R., le Sage, C., Diosdado, B., van der Waal, M., Oude Vrielink, J. A., Bolijn, A., et al. (2008). Regulation of the adenomatous polyposis coli gene by the miR135 family in colorectal cancer. Cancer Res. 68, 5795-5802. doi: 10.1158/00085472.CAN-08-0951

Nemani, M., Linares-Cruz, G., Bruzzoni-Giovanelli, H., Roperch, J. P., Tuynder, M., Bougueleret, L., et al. (1996). Activation of the human homologue of the Drosophila sina gene in apoptosis and tumor suppression. Proc. Natl. Acad. Sci. U.S.A. 93, 9039-9042. doi: 10.1073/pnas.93.17.9039

Neuhauss, S. C., Solnica-Krezel, L., Schier, A. F., Zwartkruis, F., Stemple, D. L., Malicki, J., et al. (1996). Mutations affecting craniofacial development in zebrafish. Development 123, 357-367.

Pang, R. T., Liu, W. M., Leung, C. O., Ye, T. M., Kwan, P. C., Lee, K. F., et al. (2011). miR-135A regulates preimplantation embryo development through downregulation of E3 Ubiquitin Ligase Seven In Absentia Homolog 1A (SIAH1A) expression. PLoS One 6:e27878. doi: 10.1371/journal.pone.0027878 
Pineda, R. H., Heiser, R. A., and Ribera, A. B. (2005). Developmental, molecular, and genetic dissection of INa in vivo in embryonic zebrafish sensory neurons. J. Neurophysiol. 93, 3582-3593. doi: 10.1152/jn.01070.2004

Relaix, F., Wei, X., Li, W., Pan, J., Lin, Y., Bowtell, D. D., et al. (2000). Pw1/Peg3 is a potential cell death mediator and cooperates with Siahla in p53-mediated apoptosis. Proc. Natl. Acad. Sci. U.S.A. 97, 2105-2110. doi: 10.1073/pnas. 040378897

Rovasio, R. A., and Battiato, N. L. (2002). Ethanol induces morphological and dynamic changes on in vivo and in vitro neural crest cells. Alcohol Clin. Exp. Res. 26, 1286-1298. doi: 10.1097/01.ALC.0000026102.73486.65

Schuler, M., and Green, D. R. (2005). Transcription, apoptosis and p53: catch-22. Trends Genet. 21, 182-187. doi: 10.1016/j.tig.2005.01.001

Smith, S. M. (1997). Alcohol-induced cell death in the embryo. Alcohol Health Res. World 21, 287-297.

Steventon, B., Mayor, R., and Streit, A. (2014). Neural crest and placode interaction during the development of the cranial sensory system. Dev. Biol. 389, 28-38. doi: 10.1016/j.ydbio.2014.01.021

Su, Z., Si, W., Li, L., Zhou, B., Li, X., Xu, Y., et al. (2014). MiR-144 regulates hematopoiesis and vascular development by targeting meis1 during zebrafish development. Int. J. Biochem. Cell Biol. 49, 53-63. doi: 10.1016/j.biocel.2014. 01.005

Sulik, K. K., Johnston, M. C., and Webb, M. A. (1981). Fetal alcohol syndrome: embryogenesis in a mouse model. Science 214, 936-938. doi: 10.1126/science. 6795717

Sun, H., Chen, X., Yuan, F., Liu, J., Zhao, Y., and Chen, S. Y. (2014). Involvement of seven in absentia homolog-1 in ethanol-induced apoptosis in neural crest cells. Neurotoxicol. Teratol. 46, 26-31. doi: 10.1016/j.ntt.2014.08.006

Tiedt, R., Bartholdy, B. A., Matthias, G., Newell, J. W., and Matthias, P. (2001). The RING finger protein Siah-1 regulates the level of the transcriptional coactivator OBF-1. EMBO J. 20, 4143-4152. doi: 10.1093/emboj/20.15.4143

Walker, M. B., and Kimmel, C. B. (2007). A two-color acid-free cartilage and bone stain for zebrafish larvae. Biotech. Histochem. 82, 23-28. doi: 10.1080/ 10520290701333558

Wang, K., Chen, X., Liu, J., Zou, L. P., Feng, W., Cai, L., et al. (2018). Embryonic exposure to ethanol increases the susceptibility of larval zebrafish to chemically induced seizures. Sci. Rep. 8:1845. doi: 10.1038/s41598-018-20288-2

Winter, M., Sombroek, D., Dauth, I., Moehlenbrink, J., Scheuermann, K., Crone, J., et al. (2008). Control of HIPK2 stability by ubiquitin ligase Siah-1 and checkpoint kinases ATM and ATR. Nat. Cell Biol. 10, 812-824. doi: 10.1038/ ncb1743

Wu, S., Lin, Y., Xu, D., Chen, J., Shu, M., Zhou, Y., et al. (2012). MiR-135a functions as a selective killer of malignant glioma. Oncogene 31, 3866-3874. doi: 10.1038/onc.2011.551

Xu, B., Tao, T., Wang, Y., Fang, F., Huang, Y., Chen, S., et al. (2016). hsa-miR135a-1 inhibits prostate cancer cell growth and migration by targeting EGFR. Tumour Biol. 37, 14141-14151. doi: 10.1007/s13277-016-5196-6
Yamada, Y., Hidaka, H., Seki, N., Yoshino, H., Yamasaki, T., Itesako, T., et al. (2013). Tumor-suppressive microRNA-135a inhibits cancer cell proliferation by targeting the c-MYC oncogene in renal cell carcinoma. Cancer Sci. 104, 304-312. doi: $10.1111 /$ cas.12072

Yan, D., Dong, J., Sulik, K. K., and Chen, S. Y. (2010). Induction of the Nrf2-driven antioxidant response by tert-butylhydroquinone prevents ethanol-induced apoptosis in cranial neural crest cells. Biochem. Pharmacol. 80, 144-149. doi: 10.1016/j.bcp.2010.03.004

Yan, X., Li, W., Yang, L., Dong, W., Chen, W., Mao, Y., et al. (2018). MiR-135a protects vascular endothelial cells against ventilator-induced lung injury by inhibiting PHLPP2 to activate PI3K/Akt pathway. Cell. Physiol. Biochem. 48, 1245-1258. doi: 10.1159/000492010

Yuan, F., Chen, X., Liu, J., Feng, W., Cai, L., Wu, X., et al. (2018). Sulforaphane restores acetyl-histone $\mathrm{H} 3$ binding to $\mathrm{Bcl}-2$ promoter and prevents apoptosis in ethanol-exposed neural crest cells and mouse embryos. Exp. Neurol. 300, 60-66. doi: 10.1016/j.expneurol.2017.10.020

Yuan, F., Chen, X., Liu, J., Feng, W., Wu, X., and Chen, S. Y. (2017). Up-regulation of Siah1 by ethanol triggers apoptosis in neural crest cells through p38 MAPKmediated activation of p53 signaling pathway. Arch. Toxicol. 91, 775-784. doi: 10.1007/s00204-016-1746-3

Zhang, J., Guenther, M. G., Carthew, R. W., and Lazar, M. A. (1998). Proteasomal regulation of nuclear receptor corepressor-mediated repression. Genes Dev. 12, 1775-1780. doi: 10.1101/gad.12.12.1775

Zhang, P., Wang, G., Lin, Z., Wu, Y., Zhang, J., Liu, M., et al. (2017). Alcohol exposure induces chick craniofacial bone defects by negatively affecting cranial neural crest development. Toxicol. Lett. 281, 53-64. doi: 10.1016/j.toxlet.2017. 09.010

Zhao, X., Sun, Z., Li, H., Jiang, F., Zhou, J., and Zhang, L. (2017). MiR-135a-5p modulates biological functions of thyroid carcinoma cells via targeting VCAN 3'-UTR. Cancer Biomark. 20, 207-216. doi: 10.3233/CBM-170566

Zheng, S. Y., Fu, X. B., Xu, J. G., Zhao, J. Y., Sun, T. Z., and Chen, W. (2005). Inhibition of p38 mitogen-activated protein kinase may decrease intestinal epithelial cell apoptosis and improve intestinal epithelial barrier function after ischemia- reperfusion injury. World J. Gastroenterol. 11, 656-660. doi: 10.3748/ wjg.v11.i5.656

Conflict of Interest: The authors declare that the research was conducted in the absence of any commercial or financial relationships that could be construed as a potential conflict of interest.

Copyright (c) 2020 Yuan, Yun, Fan, Li, Lu, Liu, Feng and Chen. This is an open-access article distributed under the terms of the Creative Commons Attribution License (CC BY). The use, distribution or reproduction in other forums is permitted, provided the original author(s) and the copyright owner(s) are credited and that the original publication in this journal is cited, in accordance with accepted academic practice. No use, distribution or reproduction is permitted which does not comply with these terms. 